\title{
Indicador da viabilidade de customização em massa de produtos industriais
}

\author{
Rogério Royer, M.Eng. \\ rogroyer@ppgep.ufrgs.br \\ Programa de Pós-Graduação em Engenharia de Produção (PPGEP) \\ Universidade Federal do Rio Grande do Sul (UFRGS) \\ Praça Argentina, 9, $2^{\circ}$ Andar, Sala LOPP \\ Porto Alegre, RS, 90040-020 \\ Flávio S. Fogliatto, Ph.D. \\ ffogliatto@ppgep.ufrgs.br \\ Departamento de Eng ${ }^{a}$ de Produção e Transportes (DEPROT) \\ Universidade Federal do Rio Grande do Sul (UFRGS) \\ Praça Argentina, 9, $2^{\circ}$ Andar, Sala LOPP \\ Porto Alegre, RS, 90040-020
}

\begin{abstract}
Customização em massa (CM) é um conceito criado por Davis (1989) para descrever um novo paradigma em gestão da produção, onde o objetivo é atender a demanda por produtos e serviços individualizados. A CM permite oferecer produtos customizados a preços similares aos de itens produzidos em massa. Neste artigo, propõe-se um índice que mede a viabilidade da customização em massa de produtos industriais. $\mathrm{O}$ índice proposto permite estabelecer um ranking de customização para as características de um produto; quanto melhor posicionada a característica no ranking, mais facilmente ela se adaptará à Customização em Massa. O índice proposto é operacionalizado através de uma utilização inédita da matriz do DFQ (Desdobramento da Função Qualidade) onde, através de operações matriciais, efetuam-se operações inversas às operações usuais do DFQ. Um estudo de caso é apresentado para ilustrar a técnica proposta.
\end{abstract}

Palavras-chave: índice de customização de produtos; implementação da customização em massa; DFQ.

Mass customization (MC) is a concept created by Davis (1989) to describe a new paradigm in production management that aims to fulfill the demand for tailor-made products and services. MC enables the delivery of customized products at prices similar to mass-produced items. This article proposes a customization index to estimate the viability of implementing MC systems. The index establishes a ranking of customizability for different characteristics of a product; that is based on three variables that are customer requirements, supplier delivery flexibility, and production flexibility. The MC index is implemented through an original application of the Quality Function Deployment (QFD) matrix. A case study illustrates the method proposed.

Keywords: mass customization index; mass customization implementation; QFD.

\section{Introdução}

Customização em Massa (CM) é uma nova estratégia de gestão da produção concebida por Davis (1989). O objetivo da CM é oferecer produtos ou serviços personalizados a preços similares aos de itens produzidos em massa (KAY, 1993; KOTHA, 1996). Apesar de identificada como uma importante estratégia de produção (BOYNTON et al., 1993; VAN HOEK, 2000), a implementação da CM pode ser dificultada por uma série de empecilhos técnicos e organizacionais, dentre os quais destacam-se $(i)$ existência de portfolios complexos de opções aos clientes (LIECHTY et al., 2001), (ii) proliferação de diferentes tipos de componentes e fornecedores (JIAO E TSENG, 2000), e (iii) requisitos de alta flexibilidade em processos de manufatura (McCUTCHEON et al., 1994). Para contornar tais dificuldades, empresas devem analisar seus processos produtivos, bem como as demandas de seus mercados consumidores, de forma a (ZIPKIN, 2001): (i) definir a natureza e extensão da CM que pode 
efetivamente ser oferecida aos clientes, e (ii) identificar prioridades de melhorias com vistas a implantação da CM, especialmente no que diz respeito ao projeto de produtos, modificações nos processos produtivos e gestão da cadeia de suprimentos.

Neste artigo, propõe-se um índice de customização que permite estimar a viabilidade da implementação de sistemas de CM. O índice proposto estabelece um ranking para diferentes características de um produto relativamente ao seu potencial de customização. Três variáveis são consideradas na composição do índice: demandas dos clientes, flexibilidade de entrega de partes componentes dos produtos pelos fornecedores e flexibilidade dos processos de manufatura.

O índice de customização é implementado através de uma aplicação original das matrizes do Desdobramento da Função Qualidade (DFQ). Inicialmente, itens de customização (i.e., características ou demandas do produto em estudo que possam ser personalizadas para atender a preferências dos clientes) são listados, em conjunto com seus correspondentes pesos de preferência. Itens de customização são então correlacionados com itens de projeto (i.e., partes componentes) do produto em uma matriz do DFQ. Como resultado, são obtidos pesos de importância para os itens de projeto; tais pesos são então ajustados para refletir a flexibilidade do projeto e/ou dos fornecedores dos itens de projeto. Itens de projeto e seus pesos de importância são então correlacionados com etapas do processo de manufatura do produto em uma segunda matriz do DFQ. Os pesos de importância para as etapas do processo, resultantes da análise da segunda matriz, são então ajustados para refletir o grau de flexibilidade de cada etapa. Por flexibilidade das etapas do processo, entende-se a habilidade de produção de conjuntos distintos de componentes ou subconjuntos, a partir de diferentes materiais e utilizando diferentes seqüências de operações, sem necessidade de grandes setups (BROWNE et al., 1984). Finalmente, procede-se com operações matriciais em ordem reversa àquela proposta no algoritmo do DFQ.

O uso das matrizes do DFQ na determinação de um índice de customização permite incorporar requisitos técnicos e mercadológicos em sua composição. Requisitos mercadológicos dizem respeito a demandas de clientes por variedade e personalização; requisitos técnicos dizem respeito à estrutura do produto e de seu processo de manufatura. O resultado da análise reversa das matrizes do DFQ vem dado na forma de um índice para cada item de customização, que leva em consideração pesos de preferência, relativos às demandas dos clientes, e pesos de desempenho, relativos à flexibilidade de fornecedores e de etapas do processo. Tal índice pode auxiliar na determinação $(i)$ da viabilidade técnica e financeira da customização em massa do produto em estudo, e (ii) de aspectos a serem priorizados em ações de melhoria no projeto do produto e em seu processo de manufatura, com vistas a reduzir custos e incrementar o potencial de oferta de produtos customizados.

Este artigo apresenta duas importantes contribuições para a pesquisa em CM e em DFQ. Primeiramente, o artigo oferece uma abordagem para determinar a viabilidade de implantação de estratégias de $\mathrm{CM}$ em empresas de manufatura. Tal abordagem contribui de forma significativa para a pesquisa em $\mathrm{CM}$, enfocada predominantemente em aspectos conceituais e estratégicos, em detrimento de aspectos operacionais relativos à sua implantação. O artigo também traz uma aplicação original das matrizes do DFQ e propõe um procedimento de cálculo reverso ao algoritmo usual do DFQ inédito na literatura.

Este artigo encontra-se organizado em cinco seções, incluindo a presente introdução. A seção 2 traz uma revisão teórica sobre $\mathrm{CM}$ e flexibilidade. Na seção 3, descreve-se o método proposto para determinação do índice de customização. A seção 4 traz um estudo de caso que ilustra uma aplicação prática do método proposto. A seção 5 contém as conclusões do trabalho.

\section{Customização em Massa e Flexibilidade}

A CM pode ser definida como a habilidade de projetar e manufaturar produtos ou serviços individualizados a preços similares aos de itens produzidos em massa (KOTHA, 1996; DA SILVEIRA et al., 2001). A CM é atingida através de uma abordagem made-to-order, onde clientes solicitam a manufatura e entrega de produtos individualizados, de acordo com suas especificações (LAMPEL E MINTZBERG, 1996; SOPHIE-LEE et al., 2000).

Uma série de modificações ocorridas ao longo dos anos na indústria e no mercado explicam o surgimento de sistemas de CM. Como primeiro fator, destaca-se a crescente demanda por especialização e variedade nos produtos de diferentes setores indutriais (McCUTCHEON et al., 1994). Também podem-se citar mudanças no padrão de demanda dos clientes, que induziram a uma redução nos ciclos de vida dos produtos (BOYNTON et al., 1993; Hart, 1995; KOTHA, 1995). Finalmente, avanços em tecnologias e metodologias de operações habilitaram o desenvolvimento da flexibilidade na manufatura (ADAMIDES, 1996; SONGENAGI, 1997). 
A pesquisa acadêmica sobre a Customização em Massa pode ser considerada como incipiente. Estudos sobre CM surgiram a partir de meados da década de 90, seguindo os desenvolvimentos fundamentais em Davis (1989) e Pine e Pietrocini (1993). A maioria desses estudos tiveram como foco aspectos da CM relacionados à estratégia competitiva (por exemplo, BOYNTON et al., 1993; LAMPEL e MINTZBERG, 1996) e de manufatura (por exemplo, WESTBROOK e WILLIAMSON, 1993; e Kotha, 1996). Somente mais recentemente, a pesquisa acadêmica voltou-se para abordagens operacionais de implantação da CM (por exemplo, JIAO e TSENG, 2000; LIECHTY et al., 2001), focalizando principalmente aspectos relacionados à seleção do portfolio de produtos a serem customizados. Abordagens mais abrangentes que permitam determinar o potencial de oferta de produtos customizados por sistemas de manufatura, integrando na análise elementos relacionados a produto, processo e elementos da cadeia de suprimentos estão ausentes, todavia, na literatura sobre CM.

Flexibilidade de manufatura pode ser definida como a habilidade (do sistema de manufatura) de adaptarse a mudanças e incertezas em seu ambiente (SLACK, 1987; GUPTA e GOYAL, 1989; SETHI e SETHI, 1990). Tal flexibilidade possibilita alterações no mix de produtos e processos com pequenas penalizações em termos de tempo, custo e desempenho (UPTON 1994; VAN DIJK, 1995).

Flexibilidade na produção é o principal requisito dos sistemas de CM. Uma manufatura flexível torna viável a operação em pequenos lotes, comportando uma grande variedade de produtos e componentes, como ocorre na CM (McCUTCHEON et al., 1994; ZIPKIN, 2001). Diversos autores apresentam exemplos da flexibilidade na produção como habilitadora dos sistemas de CM. Westbrook e Williamson (1993) identificam a flexibilidade de manufatura como essencial para que empresas japonesas pudessem oferecer produtos customizados a preços competitivos. Lau (1995) e Lei et al. (1996) enfatizaram o papel das tecnologias de flexibilidade na viabilização da CM. Boynton et al. (1993) identificaram a flexibilidade como essencial para que empresas pudessem atender demandas típicas da CM. Segundo Newman et al. (1993), incrementos na flexibilidade têm por objetivo proporcionar um equilíbrio entre demandas dos clientes em termos de produtos ou serviços customizados e a habilidade dos processos e fornecedores de entregar tais produtos ou serviços nos níveis de qualidade, custo e prazo desejados.

A medição do grau de flexibilidade de sistemas de manufatura não é uma tarefa trivial. O conceito de flexibilidade de manufatura é complexo e multi- dimensional; o desenvolvimento de diferentes tipos de flexibilidade tem por objetivo lidar com diferentes tipos de incerteza e variação nos ambientes internos e externos à empresa (SETHI e SETHI, 1990; DE TONI e TONCHIA, 1998). Desta forma, um dos principais aspectos abordados na literatura sobre flexibilidade de manufatura é a classificação dos tipos de flexibilidade de acordo com o objeto de variação por elas abordados. Gupta e Goyal (1989), Sethi e Sethi (1990), Ramasesh e Jayakumar (1991) e De Toni e Tonchia (1998) apresentaram revisões de literatura acerca dos diferentes tipos de flexibilidade e medidas correspondentes presentes na literatura; a Figura 1 apresenta um resumo dos resultados obtidos por estes autores.

Gupta e Goyal (1989) e Ramasesh e Jayakumar (1991) discutiram critérios auxiliares na escolha dos tipos de flexibilidade e correspondentes medidas a serem utilizados na prática. Tal escolha depende de três aspectos principais: $(i)$ a estratégia competitiva da firma, o que requer dos administradores uma correta identificação e priorização dos tipos de flexibilidade mais relevantes em cada caso; (ii) os diferentes tipos de variedade e incerteza existentes nos meios internos e externos à empresa; e (iii) a configuração do processo de manufatura, que pode permitir diferentes tipos de flexibilidade.

Do grande número de artigos que abordam o tema da flexibilidade de manufatura, poucos discutem como incorporar a flexibilidade em projetos de CM. Autores como McCutcheon et al. (1994) e Zipkin (2001), por exemplo, destacaram a necessidade da medição e desenvolvimento das capacidades de flexibilidade das firmas que têm por objetivo sistemas de CM, sem contudo oferecer métodos que permitam implementar tal análise de flexibilidade na prática.

\section{Metodologia}

\subsection{Identificação do produto a ser customizado}

Nesta etapa, é essencial que a empresa realize uma avaliação estratégica sobre o produto a ser abordado no estudo de customização. Tal produto deve apresentar $(i)$ relevância em termos de faturamento para a empresa, (ii) potencial viabilidade de customização e (iii) demanda pré-estabelecida por customização, que pode ser diretamente apontada pelos usuários do produto ou identificada como essencial para o prolongamento da vida do produto no mercado.

A análise do produto a ser customizado, em particular quanto a sua viabilidade técnica inicial, pode ser auxiliada pela revisão dos fatores internos e externos a serem considerados para a customização 
(EASTWOOD, 1996; LAU, 1995). Estudos de customização costumam resultar em adaptações e melhorias de produtos já existentes; desta forma, estudos de engenharia de valor (CSILLAG, 1995; MURY, 2000) podem auxiliar na determinação da viabilidade econômica das potenciais adaptações previstas para o produto em estudo.
A identificação correta do produto a ser customizado é essencial para o sucesso do processo de customização, já que a validade dos resultados dependerá da existência de uma demanda efetiva por customização.

\begin{tabular}{|c|c|c|}
\hline Flexibilidade & Habilidade para & Medidas \\
\hline Produto & $\begin{array}{l}\text { Introduzir e manufaturar novos } \\
\text { componentes (partes) e produtos } \\
\text { (NARASIMHAN e DAS, 1999) }\end{array}$ & $\begin{array}{l}\text { Custo e tempo para introdução de novos produtos } \\
\text { (NARASIMHAN e DAS, 1999) } \\
\text { Número de partes produzidas por ano (JAIKUMAR, 1984) }\end{array}$ \\
\hline Mix & $\begin{array}{l}\text { Manufaturar uma variedade de } \\
\text { produtos dentro de um determinado } \\
\text { período (DIXON, 1992) }\end{array}$ & $\begin{array}{l}\text { Número médio de características distintas em produtos } \\
\text { gerados no período (DIXON, 1992) } \\
\text { Tempo necessário para ajustar o mix de produtos sendo } \\
\text { manufaturado (SLACK e CORREA, 1992) }\end{array}$ \\
\hline Entrega & $\begin{array}{l}\text { Ajustar e cumprir datas ajustadas de } \\
\text { entrega (SLACK e CORREA, 1992) }\end{array}$ & $\begin{array}{l}\text { Número de dias em que as datas de entrega podem ser } \\
\text { adiantadas (SLACK e CORREA, 1992) } \\
\text { Lead time de entrega (OLHAGER, 1993) }\end{array}$ \\
\hline Produção & $\begin{array}{l}\text { Produzir economicamente um } \\
\text { conjunto de componentes (GUPTA e } \\
\text { GOYAL, 1989) }\end{array}$ & $\begin{array}{l}\text { Probabilidade de o sistema produzir um novo componente } \\
\text { com o conjunto existente de máquinas (GUPTA e GOYAL, } \\
\text { 1989) } \\
\text { Número de componentes que o sistema é capaz de } \\
\text { produzir (CHATTERJEE } \text { et al., 1984) }\end{array}$ \\
\hline Volume & $\begin{array}{l}\text { Operar de forma econômica com } \\
\text { volumes de produção distintos } \\
\text { (BROWNE } \text { et al., 1984) }\end{array}$ & $\begin{array}{l}\text { Menor volume de produção para o qual o sistema é ainda } \\
\text { rentável (BROWNE et al., 1984) } \\
\text { Média da razão entre a flutuação no volume e a capacidade } \\
\text { total (GERWIN, 1987) }\end{array}$ \\
\hline Expansão & $\begin{array}{l}\text { Construir e expandir a capacidade } \\
\text { operacional (BROWNE } \text { et al., 1984) }\end{array}$ & $\begin{array}{l}\text { Tamanho máximo que o sistema pode ter (BROWNE et al., } \\
\text { 1984) } \\
\text { Custo e tempo necessário para adicionar uma determinada } \\
\text { quantidade de capacidade (CARTER, 1986) }\end{array}$ \\
\hline Processo & $\begin{array}{l}\text { Operacionalizar trocas de produtos a } \\
\text { partir de um dado conjunto de partes } \\
\text { componentes (GUPTA e GOYAL, } \\
\text { 1989) }\end{array}$ & $\begin{array}{l}\text { Tempo e custo de setup entre diferentes pedidos } \\
\text { (GUPTA e GOYAL, 1989) } \\
\text { Tamanho dos lotes (HEIJLTJES, 2000) }\end{array}$ \\
\hline Programação & $\begin{array}{l}\text { Operar sem intervenção } \\
\text { (desatendido) durante um } \\
\text { determinado período de tempo } \\
\text { (RAMASESH e JAYAKUMAR, } \\
\text { 1991) }\end{array}$ & $\begin{array}{l}\text { Percentual esperado de tempo operacional sem } \\
\text { interrupção ao longo dos turnos (JAIKUMAR, 1984; } \\
\text { RAMASESH e JAYAKUMAR, 1991) } \\
\text { Proporção do número de equipamentos programáveis } \\
\text { frente ao número total de equipamentos (COX, 1989) }\end{array}$ \\
\hline Roteamento & $\begin{array}{l}\text { Utilizar rotas alternativas de } \\
\text { processamento na fabricação de um } \\
\text { produto (RAMASESH e } \\
\text { JAYAKUMAR, 1991) }\end{array}$ & $\begin{array}{l}\text { Número médio de maneiras através das quais uma parte } \\
\text { pode ser produzida (CHUNG e CHEN, 1989; } \\
\text { RAMASESH e JAYAKUMAR, 1991) } \\
\text { Razão entre o número existente e o número potencial de } \\
\text { ligações entre máquinas no sistema de produção (CARTER, } \\
\text { 1986) }\end{array}$ \\
\hline Máquina & $\begin{array}{l}\text { Executar diferentes operações em } \\
\text { máquinas sem que a troca de uma } \\
\text { máquina para outra implique em } \\
\text { esforços proibitivos (SETHI e SETHI, } \\
\text { 1990) }\end{array}$ & $\begin{array}{l}\text { Custo da troca entre operações (SETHI e SETHI, 1990) } \\
\text { Quantidade de diferentes inputs que a máquina pode } \\
\text { aceitar (GERWIN, 1987) }\end{array}$ \\
\hline Mão-de-obra & $\begin{array}{l}\text { Executar uma ampla variedade de } \\
\text { tarefas de maneira eficiente } \\
\text { (RAMASESH e JAYAKUMAR, } \\
\text { 1991) }\end{array}$ & $\begin{array}{l}\text { Número médio de tarefas executadas pelos trabalhadores } \\
\text { (ATKINSON, 1985; RAMASESH e JAYAKUMAR, 1991) } \\
\text { Participação de empregados em tarefas de maior } \\
\text { responsabilidade (BENSON, 1996) }\end{array}$ \\
\hline
\end{tabular}




\subsection{Escolha da ferramenta a ser utilizada na coleta de informações acerca dos elementos de customização demandada}

Nesta etapa, seleciona-se uma ferramenta auxiliar na identificação das preferências e necessidades de individualização do produto em estudo. Tais informações são coletadas, preferencialmente, junto aos usuários do produto, sendo designadas, genericamente, por elementos de customização demandada. A escolha da ferramenta apropriada para a coleta de informações nesta fase considera aspectos como disponibilidade de dados, grau de detalhamento desejado e custo de aquisição.

Para identificar elementos do produto a serem customizados conforme as preferências do consumidor, deve-se utilizar uma ferramenta que capte os desejos estabelecidos pelo cliente para a personalização do produto. Como ferramentas recomendadas listam-se: $(i)$ pesquisa de mercado (HAYES, 1998; KOTLER, 1998; CHURCHILLe PETER, 2000); (ii) utilização de dados históricos existentes na empresa (KOTLER, 1998; CHURCHILL e PETER, 2000), e (iii) coleta de informações com o pessoal especializado da empresa (ROSA, 1999).

\subsection{Identificação, classificação e quantificação dos elementos a serem customizados}

A identificação dos elementos a serem customizados é realizada através da aplicação da ferramenta definida na etapa anterior para a coleta de dados. Tal ferramenta deve associar aos elementos a serem customizados uma ponderação de importância dada pelo cliente. Nesta proposta, recomenda-se a utilização da pesquisa de mercado e coleta de informações com o pessoal especializado da empresa.

\section{A classificação dos elementos a serem} customizados em grupos que representem os aspectos do produto é importante para a elaboração posterior da árvore da customização demandada. A árvore da customização demandada organiza os elementos a serem customizados em uma estrutura hierárquica, desdobrando-os em níveis primário, secundário e terciário. Assim, a classificação dos elementos deve ser em grupos, sendo estes organizados dentro dos três níveis. O desdobramento de elementos da árvore de customização demandada em número de níveis superior a três, costuma dificultar as etapas do método, não sendo recomendado. Esta classificação deve ser feita pela equipe de trabalho da empresa que estiver realizan- do o estudo sobre customização, devendo-se aqui considerar as seguintes observações:

A) O nível primário deve ser composto por grupos que dividam o produto em aspectos gerais; por exemplo, aspectos operacionais e estruturais do produto.

B) O nível secundário deve ser composto por pequenos grupos que estabelecem uma relação com os aspectos gerais descritos no nível primário e os elementos do nível terciário; por exemplo, aspectos funcionais e estéticos do produto.

C) Os itens relacionados no nível terciário são aqueles identificados pelos clientes como elementos desejados a serem customizados.

D) Dentro de cada nível, grupos e/ou elementos devem ser mutuamente exclusivos.

A atribuição de pesos para os elementos e grupos em que eles foram distribuídos permitirá a quantificação dos elementos na elaboração da árvore da customização demandada. A atribuição de pesos aqui deve ser feita utilizando-se os dados coletados durante a identificação dos elementos a serem customizados. No final desta etapa, tem-se (i) pesos de importância, em uma escala de zero a dez, para elementos descritos no nível secundário e (ii) pesos de importância, em uma escala de zero a dez, para elementos customizáveis descritos no nível terciário.

\subsection{Elaboração da árvore da customização demandada}

A árvore da customização demandada organiza os elementos a serem customizados em três níveis, e origina uma ponderação para os elementos conforme as técnicas do DFQ (AKAO, 1990). A ponderação dos elementos a serem customizados, resultantes da árvore de customização demandada, será utilizada como valor de entrada na matriz da customização demandada, a ser vista na próxima etapa. A elaboração da árvore da customização demandada utiliza as informações obtidas na seção 3.3, com o objetivo de calcular um índice para os elementos de customização demandados através da ponderação na etapa anterior. Para tanto, seguem-se os seguintes passos:

1 Passo - Calcular o \% expresso nos níveis secundário (\%NS) e terciário $(\% N T)$, indicando a importância do item em cada nível se comparado com os demais itens da coluna. Para a obtenção destes percentuais, utilizam-se respectivamente as equações (1) e (2), onde $P_{N S}$ é o peso do item do nível secundário, $S P N S$ é o somatório dos pesos dos itens do nível secundário, $P_{N T}$ é o peso do item 
do nível terciário e SPSNT é o somatório dos pesos dos itens do subconjunto no nível terciário ao qual o item pertence.

$$
\begin{aligned}
& \% \mathrm{NS}=\frac{\mathrm{P}_{\mathrm{NS}}}{\mathrm{SPNS}} \\
& \% \mathrm{NT}=\frac{\mathrm{P}_{\mathrm{NT}}}{\mathrm{SPSNT}}
\end{aligned}
$$

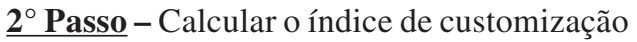
demandada $(I C D)$, que indica a importância dos elementos relacionados no nível terciário. Aqui é expressa a importância que o cliente atribui aos elementos do produto, propostos para customização. Para o cálculo do $I C D$, utiliza-se a equação (3).

$$
\mathrm{ICD}=\% \mathrm{NS} \times\left(\frac{\% \mathrm{NT}}{100}\right)
$$

$\underline{3^{\circ} \text { Passo }}$ - Calcular o índice de customização demandada corrigido $\left(I C D^{*}\right)$, que indica a importância dos elementos relacionados no nível terciário, considerando diferenças existentes entre subgrupos do nível terciário (que ocorrem quando o número de elementos em cada subgrupo não é igual). Para o cálculo do $I C D^{*}$, utiliza-se a equação (4), onde $P_{N S}$ é o peso do item no nível secundário; $I C D$ é o índice de customização demandada do item e $I C D_{\max / s u b}$ é o valor máximo entre os $I C D$ dos elementos do subgrupo.

$$
\mathrm{ICD}^{*}=\mathrm{P}_{\mathrm{NS}} \times\left(\frac{\mathrm{ICD}}{\mathrm{ICD}_{\text {max./sub }}}\right)
$$

\subsection{Elaboração da matriz da customização demandada}

A matriz da customização demandada é construída a partir do desdobramento dos elementos de customização demandada e dos itens de projeto. Itens de projeto são os elementos (peças, componentes, conjuntos, subconjuntos etc.) que formam o produto.

Na matriz de customização demandada, conforme a Figura 2, as linhas são formadas pelos elementos de customização demandados e seus respectivos $I C D^{*}$; as colunas da matriz, por sua vez, são formadas pelos itens de projeto. Os demais elementos que compõem a matriz serão explicados ainda nesta etapa. A elaboração da matriz da customização demandada tem como objetivo a priorização dos itens de produto conforme a importância que estes apresentam e a intensidade de seu relacionamento com os elementos de customização demandados pelos clientes. A sua elaboração é realizada através dos seguintes passos:

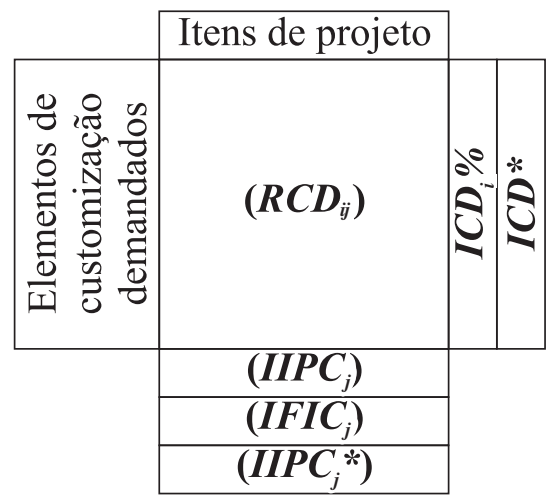

Figura 2 - Estrutura genérica da matriz da customização demandada

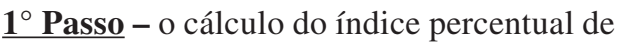
priorização dos elementos de customização demandados $\left(I C D_{i} \%\right)$ é feito utilizando-se $I C D^{*}$. O objetivo é apresentar o $I C D^{*}$ em formato percentual. Os elementos de customização demandados nas linhas da matriz são designados por $i=1,2, \ldots ., I$.

$\underline{2^{\circ} \text { Passo }}$ - relacionamentos existentes entre os elementos de customização demandados e os itens de projeto são identificados no cruzamento das linhas com as colunas, no corpo da matriz na Figura 1. Itens de projeto nas colunas da matriz são designados por $j=1,2, \ldots, J$. A intensidade dos relacionamentos entre os elementos de customização e os itens de projetos $\left(R C D_{i j}\right)$ é expressa utilizando a escala da Tabela 1.

Tabela 1 - Escala de intensidades de relacionamentos na matriz da customização demandada e de processos

\begin{tabular}{cccccc}
\hline \multicolumn{2}{c}{ Intensidade do relacionamento } & Forte & Médio & Fraca & Inexistente \\
\cline { 1 - 2 } Matriz da customização demandada & $R C D_{i j}$ & 5,0 & 3,0 & 1,0 & 0,0 \\
\hline Matriz de processos & $R E P_{k j}$ & & & & \\
\hline
\end{tabular}


$\underline{3^{\circ} \text { Passo }}$ - o cálculo da importância dos itens de projeto para a customização $\left(I I P C_{j}\right)$ considera o índice $I C D_{i} \%$ e a relação entre cada elemento a ser customizado e os itens de projeto $\left(R C D_{i j}\right)$, através da equação:

$I I P C_{j}=\sum_{i=1}^{I} I C D_{i} \% \times R C D_{i j}, j=1, \ldots, J$.

$\underline{4^{\circ} \text { Passo }}$ - para o cálculo do índice de flexibilidade dos itens comprados $\left(I F I C_{j}\right)$ deve-se, primeiramente, identificar os tipos de flexibilidade relacionados aos itens de projeto do produto em estudo. Na literatura são descritos alguns tipos de flexibilidade que podem ser utilizados para identificar a capacidade que os fornecedores têm em atender as necessidades da cadeia produtiva (SETHI e SETHI, 1990; RAMASESH e JAYAKUMAR, 1991; BARAD N NOF, 1997; SHEWCHUK E MOODIE, 1998; NARASIMHANe DAS, 1999; PARKER e WIRTH, 1999).

Após selecionar-se os indicadores de flexibilidade a serem utilizados, deve-se avaliar os itens de projeto com relação às formas de medida escolhidas, tal que a soma das ponderações some 1. Uma ponderação final (soma das ponderações atribuídas para os indicadores de flexibilidade escolhidos) entre 0 e 1 será definida para os itens de projeto comprados. Tal ponderação será o índice de flexibilidade dos itens comprados $\left(I F I C_{j}\right)$, que deverá identificar a facilidade com a qual um determinado item de projeto é fornecido. Para os itens não comprados externamente, podese atribuir ao $I F I C_{j}$ o valor 1 , conferindo-lhe uma flexibilidade máxima (ou seja, supõe-se total controle da empresa sobre os itens).

Itens comprados com baixa flexibilidade terão seus $I I P C_{j}$ reduzidos através da seguinte equação (onde $I I P C_{j}^{*}$

$I I P C_{j}^{*}=I I P C_{j} \times I F I C_{j} ; j=1,2, \ldots, J$.

denota a importância corrigida dos itens de projeto):

Um valor zero de $I I P C_{j}^{*}$ indicará que o item apresenta-se como inviável quanto à sua capacidade de customização, dificultando, consequentemente, a customização de todos os elementos a ele relacionados.

\subsection{Elaboração da matriz de processos}

A matriz de processos é construída a partir do desdobramento das etapas do processo de fabricação do produto, associadas aos itens de projeto listados na etapa anterior. Na matriz de processos, conforme Figura 3, as colunas são formadas pelos itens de projeto e seus respectivos $I I P C_{j}{ }_{j}^{*}$, e as linhas são formadas pelas etapas do processo. Os demais elementos que compõem a matriz de processos serão explicados no decorrer desta etapa. A utilização da matriz de processos tem como objetivo identificar os processos críticos para customização do produto, candidatos naturais à otimização.

Estes são os passos para a construção da matriz de processos:

$\underline{\mathbf{1}^{\circ} \text { Passo }}$ - relacionamentos existentes entre as etapas do processo e itens de projeto são identificados nos cruzamentos entre linhas e colunas no corpo da matriz, conforme Figura 3. A intensidade desses relacionamentos, $R E P_{k j}$, é expressa utilizando a escala na Tabela 1. Aqui, as etapas do processo nas linhas da matriz são designadas por $k=1,2, \ldots, K$.

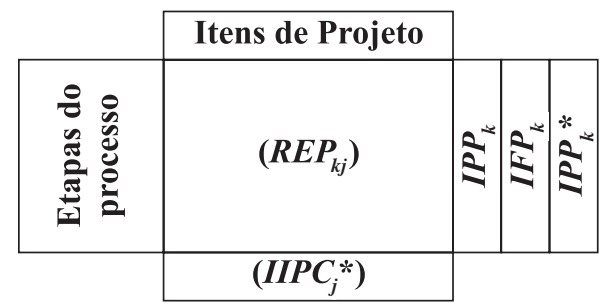

Figura 3 - Estrutura genérica da matriz de processos

$I P P_{k}=\sum_{j=1}^{J} I I P C_{j}^{*} \times R E P_{k j}, k=1,2, \ldots, K$.

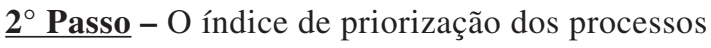
para a customização $\left(I P P_{k}\right)$ é calculado utilizani se as informações em $I I P C_{j}^{*}$ e $R E P_{k j}$, através di seguinte equação:

$\underline{\mathbf{3}^{\circ} \text { Passo }}$ - Para o cálculo do índice de flexibilidade dos processos $\left(I F P_{k}\right)$, deve-se primeiramente identificar os tipos de flexibilidade, relativos às etapas de processo, relevantes e mensuráveis (SETHI e SETTHI, 1990; RAMASESH e JAYAKUMAR, 1991; BARAD e NOF, 1997; SHEWCHUK e MOODIE, 1998; NARASIMHAN e DAS, 1999). Cada forma de medida relacionada aos tipos de flexibilidade existentes deve ser ponderada nas etapas do processo, devendo esta ponderação ser transformada em valores no intervalo entre 0 e 1 . Caso seja utilizada mais de uma forma de medida de flexibilidade para avaliar as etapas de processo, estas devem ser ponderadas em importância de forma a totalizar $100 \%$.

$\underline{4^{\circ} \text { Passo }-O ~ i ́ n d i c e ~ c o r r i g i d o ~ d e ~ p r i o r i z a c ̧ a ̃ o ~ d o s ~}$ processos para customização $\left(I P P_{k}^{*}\right)$ corrige o índice $I P P_{k}$ utilizando o índice $I F P_{k}$, através da equação:

$I P P_{k}^{*}=I P P_{k} \times I F P_{k} ; k=1,2, \ldots, K$. 


\subsection{Cálculo do índice de customização demandada considerando as condições de produção existentes}

O cálculo do índice da customização demandada considerando as condições de produção existentes $\left(I C D 2_{i}\right)$ representa uma nova ponderação para os elementos da customização demandada, utilizada em substituição à ponderação definida por $I C D_{i}^{*}$. $\mathrm{O}$ índice $I C D_{i}^{*}$ considera apenas a importância de customização atribuída pelos clientes; o índice $I C D 2$, considera também as condições de produção existentes (flexibilidade dos fornecedores dos itens comprados e das etapas de processo).

Para obter o ICD 2 realizam-se operações inversas das operações usuais na matriz do DFQ. A operação inversa da matriz do DFQ constitui-se da obtenção dos dados de entrada da matriz a partir do conhecimento dos seus resultados. Evidentemente, se os resultados não forem alterados na matriz, a operação inversa resgatará os dados de entrada originais. Modificações nos resultados, todavia, refletirão em modificações nos dados de entrada, o que é o nosso objetivo.

Na exposição que se segue, matrizes são identificadas por letras maiúsculas em negrito (por exemplo, M) e seus transpostos por $\mathbf{M}^{\mathbf{T}}$; vetores são identificados por letras minúsculas em negrito (por exemplo, v) e seus transpostos por $\mathbf{v}^{\mathbf{T}}$.

No DFQ, os valores de entrada da matriz são multiplicados pelos relacionamentos existentes dentro do corpo da matriz originando os resultados finais. A álgebra utilizada nas matrizes do DFQ é a multiplicação de um vetor (dados de entrada) por uma matriz (formada pelos relacionamentos na matriz do DFQ), o que resulta em outro vetor (resultado final do DFQ).

A álgebra utilizada nas matrizes do DFQ é apresentada na equação (9), onde e é o vetor que contém os dados de entrada da matriz, $\mathbf{M}^{\mathrm{T}}$ é a transposta da matriz de relacionamentos do DFQ e $\mathbf{s}$ é o vetor de resultados finais da matriz do DFQ. Para encontrar e a partir de M e s, é necessário determinar a pseudoinversa de M dada pela equação (10) (Strang, 1988), onde $\left(\mathbf{M} \times \mathbf{M}^{\mathbf{T}}\right)^{-1}$ é inversa de $\left(\mathbf{M} \times \mathbf{M}^{\mathbf{T}}\right)$.

$$
\begin{gathered}
\mathbf{M}^{\mathbf{T}} \times \mathbf{e}=\mathbf{s} \\
\mathbf{e}=\left(\mathbf{M} \times \mathbf{M}^{\mathrm{T}}\right)^{-1} \times \mathbf{M} \times \mathbf{s}
\end{gathered}
$$

As operações para a obtenção do $I C D 2_{i}$ são:

Primeira Operação: o objetivo aqui é calcular o índice de priorização dos itens de projeto, corrigido de forma a refletir o grau de flexibilidade apresentado pelas etapas do processo $\left(I I P C 2_{j}\right)$. O cálculo do $I I P C 2_{j}$ é feito através da operação inversa do DFQ, realizada na matriz de processos. Assim, encontram-se valores para o IIPC2 $2_{j}$ que, ao serem utilizados como valores de entrada na matriz de processos, resultarão nos valores do $I P P_{k}^{*}$, conforme a equação (11), onde ipp é o vetor formado pelos $I P P_{k}^{*}, \mathbf{P}$ é a matriz de relacionamentos entre etapas do processo e itens de projeto e iipc2 é o vetor formado pelos $I I P C 2_{j}$.

$$
\mathbf{P} \times \text { iipc2 }=\text { ipp }
$$

Calculando-se o vetor iipc2, determinam-se os valores atribuídos aos $I I P C 2_{j}$ para os itens de projeto. A partir das equações 9,10 e 11, por analogia, tem-se a solução para o cálculo do iipc2, dada por:

$$
\text { iipc2 }=\left(\mathbf{P}^{\mathrm{T}} \times \mathbf{P}\right)^{-1} \times \mathbf{P}^{\mathrm{T}} \times \text { ipp }
$$

Segunda Operação: O objetivo aqui é calcular o índice de customização demandada corrigido para refletir as condições de produção existentes $\left(I C D 2_{i}\right)$. Analogamente à primeira operação, o cálculo do $I C D 2_{i}$ é feito através da operação inversa do DFQ, utilizando-se agora a matriz da customização demandada. Assim, ao utilizarem-se os valores encontrados para o $I C D 2{ }_{i}$ como dados de entrada na matriz da customização demandada, teremos como resultado os valores de IIPC2, conforme equação (13), onde icd 2 é o vetor formado pelos $I C D 2_{i}$ e $\mathbf{C}^{\mathrm{T}}$ é a matriz transposta de relacionamentos entre elementos da customização demandada e itens de projeto.

$$
\mathrm{C}^{\mathrm{T}} \times \text { icd2 }=\text { iipc2 }
$$

Através do cálculo do vetor icd2, determinam-se os valores atribuídos aos $I C D 2$ para os elementos de customização demandada. $\mathrm{O}$ vetor icd2 é dado por:

$$
\text { icd } 2=(C \times C)^{-1} \times C \times \text { iipc2 }
$$

Os valores de $I C D 2{ }_{i}$ representam o impacto dos índices de flexibilidade sobre os pesos originais dos elementos de customização demandada. De maneira consistente, sempre que uma etapa de processo for pouco flexível, penalizam-se todos os itens de projeto a ela associada através da diminuição de seus pesos de importância; o mesmo irá ocorrer com a flexibilidade dos itens de projeto sobre os elementos de customização demandada. 


\subsection{Interpretação dos resultados}

Nesta etapa, deve-se apresentar os resultados de forma simples e clara, visando agilizar a sua leitura e interpretação. Sugere-se que sejam comparados os rankings dos elementos de customização demandados obtidos a partir de $\mathrm{ICD}_{i}^{*}$ e ICD2 $2_{i}$. Através desta comparação, será possível observar quais elementos ganharam e perderam colocações no ranking; o ganho ou perda de colocações estará ligado diretamente à flexibilidade dos itens de projeto e/ou a flexibilidade das etapas de processo.

A interpretação dos resultados realizada na etapa anterior permite que se formulem conclusões a respeito do produto a ser customizado e de sua relação com as condições de produção existentes. As conclusões devem conter, no mínimo, uma lista dos elementos prioritários a serem customizados. Informações sobre quais as modificações são necessárias no processo produtivo para atender às expectativas de customização dos clientes também devem ser registradas.

\section{Estudo de Caso}

O estudo de caso foi realizado em uma empresa de equipamentos eletrodomésticos no estado do Rio Grande do Sul. O produto analisado foi um equipamento de ar-condicionado tipo janela. Os resultados da aplicação da metodologia no estudo de caso vêm descritos a seguir.

\subsection{Identificação do produto a ser customizado}

A escolha do produto para a realização do estudo aqui apresentado foi motivada pela existência de um trabalho, realizado pelo departamento de marketing da empresa, onde se havia identificado uma demanda de mercado relativamente à customização de aparelhos de ar-condicionado tipo janela.

\subsection{Escolha da ferramenta para coleta de informações sobre a customização demandada}

Nesta etapa decidiu-se que a ferramenta mais adequada seria a utilização de dados históricos existentes na empresa pois, ao se iniciar o estudo de caso, já havia dados coletados pelo setor de marketing acerca da customização demandada pelos clientes do produto.

\subsection{Identificação, classificação e quantificação dos elementos a serem customizados}

A identificação dos elementos a serem customizados foi realizada pelo departamento de marketing da empresa. A classificação dos elementos a serem customizados foi realizada pela equipe de trabalho do departamento de Engenheira de Produto, sendo feita em três níveis. No primeiro nível foram destacados os aspectos gerais do produto, ou seja, aspectos relacionados à operação e à estrutura do equipamento de arcondicionado tipo janela. No segundo nível foram classificados aspectos do produto. Os aspectos relacionados à operação do produto foram divididos em aspectos relacionados ao desempenho e especificação e aspectos funcionais. Os aspectos relacionados à estrutura do produto foram divididos em aspectos opcionais de instalação, aspectos estéticos, aspectos estruturais e aspectos de conservação. No terceiro nível, os elementos de customização demandada, identificados na etapa anterior, foram relacionados de forma coerente com os grupos do nível secundário.

A atribuição de pesos para os elementos a serem customizados, assim como para os grupos em que eles foram distribuídos, foi elaborada pela equipe de trabalho do departamento de Engenheira de Produto. Como nos dados históricos utilizados para identificar os elementos da customização demandada não havia uma atribuição de pesos que identificasse a importância atribuída pelos clientes, houve a necessidade de se realizar uma segunda coleta de dados. Para esta segunda coleta de dados, optou-se pela coleta de informações com o pessoal especializado da empresa. Esta escolha foi realizada devido: (i) à existência de um conhecimento apurado sobre as exigências do cliente por parte dos componentes do grupo de trabalho e ( $\mathrm{ii}$ ) ao pouco tempo existente para a realização de uma pesquisa de mercado adequada.

\subsection{Elaboração da árvore da customização demandada}

A estrutura final da árvore da customização demandada para o produto vem apresentada na Tabela 2.

A partir dos dados na Tabela 2, observa-se que os três elementos mais valorizados pelos clientes para a customização do aparelho de ar-condicionado tipo janela são (i) a escolha do nível de ruído, (ii) a escolha da marca $e$ (iii) o tipo de eficiência do aparelho. Os itens menos valorizados são (i) a escolha do tipo de circulação de ar, (ii) o tipo de gás (iii) e a opção de caixa de proteção. 
Tabela 2 - Árvore da customização demandada

\begin{tabular}{|c|c|c|c|c|c|c|c|}
\hline \multirow{2}{*}{$\begin{array}{c}\text { Nível } \\
\text { Primário }\end{array}$} & Nível Secundário & $\%$ & \multirow{2}{*}{ Nível Terciário } & \multirow{2}{*}{ Peso } & \multirow{2}{*}{$\% N T$} & \multirow{2}{*}{$I C D$} & \multirow{2}{*}{$I C D^{*}$} \\
\hline & Peso & $N S$ & & & & & \\
\hline & \multirow{4}{*}{$\begin{array}{l}\text { Desempenho e } \\
\text { Especificação }\end{array}$} & \multirow[b]{5}{*}{22,1} & Tipo de Eficiência do aparelho & 8,0 & 30,0 & 6,6 & 7,2 \\
\hline & & & Escolha do nível de ruído & 10,0 & 37,6 & 8,3 & 9,0 \\
\hline & & & Escolha da Tensão & 4,3 & 16,2 & 3,6 & 3,9 \\
\hline & & & Escolha da Freqüência & 4,3 & 16,2 & 3,6 & 3,9 \\
\hline & 9,0 & & Total & 26,6 & 100 & 22,1 & \\
\hline & \multirow{5}{*}{ Aspectos funcionais } & \multirow[b]{6}{*}{16,5} & Escolha tipo de Circulação de Ar & 4,0 & 13,8 & 2,3 & 3,5 \\
\hline & & & Escolha da Versão (quente/ Frio) & 7,0 & 24,1 & 4,0 & 6,1 \\
\hline & & & Tipo de controle & 7,7 & 26,6 & 4,4 & 6,7 \\
\hline & & & Opção por kit de tratamento do ar & 6,3 & 21,7 & 3,5 & 5,5 \\
\hline & & & Tipo de Gás & 4,0 & 13,8 & 2,3 & 3,5 \\
\hline & 6,7 & & Total & 29,0 & 100 & 16,5 & \\
\hline & \multirow{4}{*}{$\begin{array}{l}\text { Opcionais de } \\
\text { instalação }\end{array}$} & \multirow[b]{5}{*}{13,0} & Opção de Moldura & 4,7 & 24,2 & 3,2 & 4,2 \\
\hline & & & Opção de Caixa de Proteção & 4,0 & 20,6 & 2,6 & 3,5 \\
\hline & & & Opção de Mangueira do dreno & 6,0 & 31,0 & 4,0 & 5,3 \\
\hline & & & Opção de Aprov. água condensado & 4,7 & 24,2 & 3,2 & 4,2 \\
\hline & 5,3 & & Total & 19,4 & 100 & 13,0 & \\
\hline & \multirow{4}{*}{ Aspectos estéticos } & \multirow[b]{5}{*}{19,7} & Escolha da Cor & 7,0 & 26,3 & 5,2 & 6,7 \\
\hline & & & Posição do Rabicho & 5,7 & 21,3 & 4,2 & 5,5 \\
\hline & & & Saída de filtro & 5,7 & 21,3 & 4,2 & 5,5 \\
\hline & & & Escolha da Marca & 8,3 & 31,1 & 6,1 & 8,0 \\
\hline & 8 & & Total & 26,7 & 100 & 19,7 & \\
\hline & \multirow{2}{*}{$\begin{array}{l}\text { Aspectos } \\
\text { Estruturais }\end{array}$} & \multirow[b]{3}{*}{14,0} & Tipo de Gabinete & 6,7 & 60,9 & 8,5 & 5,7 \\
\hline & & & Tipo de Aleta & 4,3 & 39,1 & 5,5 & 3,7 \\
\hline & 5,7 & & Total & 11,0 & 100 & 14,0 & \\
\hline & Conservação & \multirow[b]{2}{*}{14,7} & Escolha da Embalagem & 6,0 & 100 & 14,7 & 6,0 \\
\hline & 6 & & Total & 6,0 & 100 & 14,7 & \\
\hline TOTAL & 40,7 & 100 & & & & & \\
\hline
\end{tabular}




\subsection{Elaboração da Matriz da Customização Demandada}

Primeiramente, relacionaram-se os 20 elementos de customização demandados na Tabela 2 no cabeçalho das linhas da matriz e os itens de projeto no cabeçalho das colunas da matriz. Os itens de projeto do produto foram identificados pela equipe de trabalho da Engenheira de Produto, totalizando 21 componentes. Após o preenchimento das linhas e colunas da matriz, foram realizados os passos:

$\underline{\mathbf{1}^{\circ} \text { Passo }}$ - Acrescentou-se à matriz os valores do $I C D_{i} \%$ correspondente a cada um dos elementos de customização demandados, calculados a partir do $I C D^{*}$ obtido na árvore da customização demandada (Tabela 2).

$\underline{\mathbf{2}^{\circ} \text { Passo }}$ - Foram identificadas e avaliadas as intensidades dos relacionamentos existentes na matriz entre elementos de customização demandados e itens de projeto. Na avaliação da intensidade foi utilizada a escala na Tabela 2. Valores resultantes foram escritos nos cruzamentos entre linhas e colunas, no corpo da matriz.

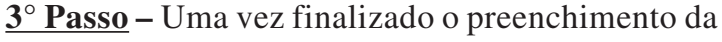
matriz, calculou-se os valores de $I I P C_{j}$, indicando a importância dos itens de projeto para a

customização; para tanto, utilizou-se a equação (5). Alguns dos resultados obtidos vêm apresentados na Tabela 3.

$\underline{4^{\circ} \text { Passo }}$ - Foi avaliada a flexibilidade de compra dos itens de projeto através do cálculo do $I F I C_{i}$. Dentre os tipos de flexibilidade descritos na literatura, a que mais se associa à compra de itens de projeto é a flexibilidade de fornecimento. Entre as características de flexibilidade, para avaliar os fornecedores dos itens de projeto, utilizou-se: (i) tempo para atendimento de pedidos $\left(I F I C 1_{j}\right)$, com peso de importância na composição do indicador de flexibilidade total do item fixado em 0,6; (ii) quantidade mínima de fornecimento estabelecida pelo fornecedor para a aceitação do pedido de compra (IFIC2 2 ), com peso de importância na composição do indicador de flexibilidade total do item fixado em 0,4 .

Para expressar a flexibilidade total dos itens de projeto

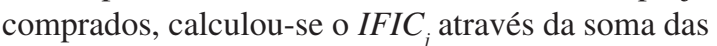
parcelas de flexibilidade, conforme equação (15). Os valores finais do $I F I C_{j}$ vêm apresentados na Tabela 3.

$$
I F I C_{j}=I F I C 1_{j}+I F I C 2_{j}
$$

$\underline{5^{\circ} \text { Passo }}$ - Nesta etapa, obteve-se o $I I P C_{j}$ *, que indica a importância corrigida dos itens de projeto para a customização, através da equação (6); resultados parciais vêm apresentados na Tabela 3 . Através do $I I P C_{j}{ }^{*}$, pode-se observar que os itens de projeto mais importantes para atender à customização do produto em estudo são $(i)$ o CJ frente plástica, (ii) o CJ condensador e (iii) o CJ evaporador; em contrapartida, os itens de projeto que menos influenciam no atendimento da customização são $(i)$ o protetor térmico, $(i i)$ o emblema do gabinete e (iii) o gás refrigerante.

\subsection{Elaboração da Matriz de Processos}

Inicialmente, foram analisadas e identificadas as etapas de processo compreendidas na fabricação do

Tabela 3 - Valores dos índices relacionados aos itens de projeto do equipamento em estudo

\begin{tabular}{c|l|c|c|c}
\hline Item & Descrição & $\boldsymbol{I I P C}_{\boldsymbol{j}}$ & $\boldsymbol{I F I C}_{\boldsymbol{j}}$ & IIPC $_{\boldsymbol{j}}^{*}$ \\
\hline 1 & Gás Refrigerante & 0,36 & 0,58 & 0,21 \\
\hline 2 & CJ Compressor & 1,00 & 0,89 & 0,89 \\
\hline 3 & CJ base & 0,42 & 1,00 & 0,42 \\
\hline 4 & CJ embalagem & 0,58 & 0,50 & 0,29 \\
\hline 5 & CJ acessórios de Instalação & 0,44 & 0,91 & 0,40 \\
\hline$\vdots$ & & $\vdots$ & $\vdots$ & $\vdots$ \\
\hline 18 & Tampa concha superior & 0,38 & 0,91 & 0,35 \\
\hline 20 & Tampa concha superior cond. & 0,45 & 0,98 & 0,44 \\
\hline 21 & Protetor térmico & 0,07 & 0,55 & 0,04 \\
\hline
\end{tabular}


produto. Foi definido como de interesse para a empresa a análise detalhada do impacto da customização sobre a linha de montagem; tais processos foram descritos detalhadamente, resultando em 47 etapas. Processos de estamparia e aletado foram agrupados em duas etapas distintas. $\mathrm{Na}$ construção da matriz de processos, foi realizada uma série de atividades, a saber:

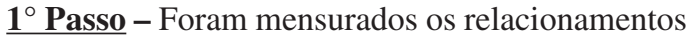
existentes entre etapas de processos e itens de projeto $\left(R E P_{k j}\right)$, usando a escala na Tabela 1.

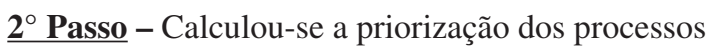
para a customização $\left(I P P_{k}\right)$ conforme a equação (7). Valores de $I P P_{k}$ encontrados para algumas etapas do processo são apresentados na Tabela 4 .

$\underline{3^{\circ} \text { Passo }}$ - Calculou-se o índice de flexibilidade dos processos $\left(I F P_{k}\right)$, para as 49 etapas selecionadas. Foi identificado pela equipe de trabalho o tempo de setup como característica mais apropriada para medir a flexibilidade das etapas de processo. Entre as várias formas de classificação existentes na literatura, o tempo de setup é classificado como uma medida de flexibilidade de resposta (BARAD e NOF, 1997) e também como flexibilidade de mix de produtos (SLACK, 1993). Por ser a única medida selecionada para quantificar a flexibilidade das etapas do processo, o tempo de setup recebeu um peso de importância igual a 1,0.

$\mathrm{Na}$ análise das etapas do processo, observou-se que apenas 5 etapas apresentavam tempos de setup diferentes de zero. Isto ocorre porque a troca de modelos na linha de produção gera modificações nas tarefas a serem executadas pelos operadores, mas não no preparo de equipamentos e/ou ferramentas. O valor do $I F P_{k}$ igual a zero foi atribuído ao processo com pior desempenho de flexibilidade, enquanto valores de $I F P_{k}$ igual a 1 foram atribuídos aos processos com maior flexibilidade. A Tabela 4 apresenta a descrição de algumas das etapas do processo e seus respectivos $I F P_{k}$.

$4^{\circ}$ Passo - Como último passo da elaboração da matriz de processos, foi calculado o índice corrigido de priorização dos processos para customização $\left(I P P_{k}^{*}\right)$, através da equação (8); resultados parciais vêm apresentados na Tabela 4. Através do cálculo do $I P P_{k}^{*}$, pode-se observar que as etapas de processo mais importantes para atender à customização do equipamento em estudo são: (i) o processo de posicionar a concha inferior do condensador, (ii) o Burst Test/Hipot e (iii) o posicionamento do CJ motor/hélice/turbina/capacitor/tampa. As etapas de processo que menos influenciam no atendimento da customização são: (i) o processo de colocar tampões, (ii) o posicionamento do rabicho no calço direito e (iii) a leitura do código de barras.

\subsection{Cálculo do índice da customização demandada incorporando as condições de produção existentes}

O cálculo do $I C D 2_{i}$ foi realizado a partir dos resultados obtidos na matriz de processos para o $I P P_{k}^{*}$. As duas operações utilizadas para a obtenção do $I C D 2$ estão descritas na seção 3.7. Os resultados do $I C D 2_{i}$ encontrados para os elementos de customização demandados estão apresentados na Tabela 5.

Na nova priorização dos elementos de customização demandados, a partir dos valores de $I C D 2_{i}$, temos

Tabela 4 - Valores dos índices relacionados a algumas etapas do processo do equipamento de ar-condicionado tipo janela

\begin{tabular}{|c|c|c|c|c|}
\hline Etapa & Descrição & $I P P_{k}$ & $I F P_{k}$ & $I P P_{k}^{*}$ \\
\hline 3 & Posicionar concha inferior condensador & 18,5 & 1,0 & 18,5 \\
\hline 18 & $\begin{array}{l}\text { Posicionar conjunto: } \\
\text { motor/hélice/turbina/capacitor/tampa }\end{array}$ & 16,1 & 1,0 & 16,1 \\
\hline 25 & Burst Test/Hipot & 16,1 & 1,0 & 16,1 \\
\hline 27 & Carga de gás & 11,2 & 0,996 & 11,12 \\
\hline 35 & Colocar tampões & 0,6 & 1,0 & 0,6 \\
\hline 41 & Encaixar calços de EPS pré-montados & 7,8 & 0,996 & 7,79 \\
\hline 42 & Posicionar rabicho no calço direito & 0,9 & 1,0 & 0,9 \\
\hline 43 & Embalar aparelho & 8,5 & 0,996 & 8,50 \\
\hline 47 & Leitura do código de barras & 0,9 & 1,0 & 0,9 \\
\hline 48 & Estamparia & 2,1 & 0,750 & 1,7 \\
\hline 49 & Aletado RAC & 9,4 & 0,000 & 4,7 \\
\hline
\end{tabular}


como elementos viáveis: $(i)$ posição do rabicho, $(i i)$ opção de moldura e (iii) opção por kit de tratamento de ar. Os elementos de customização $(i)$ opção de caixa de proteção, (ii) saída de filtro e (iii) escolha da freqüência apresentam-se como menos viáveis.

\subsection{Interpretação dos resultados}

No estudo de customização para o equipamento de arcondicionado tipo janela, foram obtidos os seguintes resultados: (i) definição dos elementos prioritários para a customização do produto $\left(I C D_{i}^{*}\right)$, (ii) definição da importância dos itens de projeto para a customização demandada ponderado pela flexibilidade dos itens comprados $\left(I I P C_{j}^{*}\right)$, (iii) priorização das etapas do processos para a customização demandada ponderado pela flexibilidade das etapas de processo $\left(I P P_{k}^{*}\right),(i v)$ priorização dos itens de projeto para a customização demandada corrigido de forma a refletir o grau de flexibilidade apresentado pelas etapas do processo $\left(I I P C 2_{j}\right)$ e $(v)$ definição dos elementos prioritários para a customização do produto, incorporado das condições de produção existentes $\left(I C D 2_{i}\right)$.

Através da Tabela 6 , foi comparado o $I C D_{i}^{*}$, que apresenta a priorização dos elementos conforme demanda do mercado, com o $I C D 2_{i}$, que considera as condições atuais de produção. Nesta análise, foi observada a diferença entre o ranking dos elementos a serem customizados obtida pelos dois índices. A diferença observada indica se as condições atuais de produção estão viabilizando a customização dos elementos indicados pela demanda de mercado. Vê-se, por exemplo, que o elemento Tipo de Gás subiu doze posições, o que indica que as condições de produção atuais favorecem a sua customização.

Tabela 5 - Resultados encontrados no cálculo do $I C D 2_{i}$ para os elementos da customização demandada

\begin{tabular}{|c|c|}
\hline Elementos da customização demandada & $\mathrm{ICD2}_{i}$ \\
\hline Tipo de Eficiência do aparelho & $-0,23$ \\
\hline Escolha do nível de ruído & 0,25 \\
\hline Escolha da Tensão & 0,22 \\
\hline Escolha da Freqüiência & $-0,49$ \\
\hline Escolha do tipo de Circulação de Ar & 0,21 \\
\hline Escolha da Versão (quente/frio) & 0,38 \\
\hline Tipo de controle & $-0,38$ \\
\hline Opção por kit de tratamento do ar & 1,11 \\
\hline Tipo de Gás & 0,23 \\
\hline Opção de Moldura & 2,23 \\
\hline Opção de Caixa de Proteção & $-7,34$ \\
\hline Opção de Mangueira do dreno & $-0,21$ \\
\hline Opção de Aproveitamento da água do condensado & 0,12 \\
\hline Escolha da Cor & 0,00006 \\
\hline Posição do Rabicho & 2,39 \\
\hline Saída de filtro & $-1,46$ \\
\hline Escolha da Marca & $-0,22$ \\
\hline Tipo de Gabinete & $-0,05$ \\
\hline Tipo de Aleta & 0,05 \\
\hline Escolha da Embalagem & 0,70 \\
\hline
\end{tabular}


A partir dos resultados obtidos, podemos concluir que:

- A empresa estudada deve estabelecer como meta realizar modificações estruturais no processo de produção existente, de forma a atender às priorizações de customização estabelecidas pelo mercado. A eficiência das modificações pode ser mensurada através do recálculo do $I C D 2$; sempre que o índice resultar igual ao $I C D_{i}^{*}$, as condições

\section{Conclusões}

No estudo realizado, propõe-se um índice de customização que mede a viabilidade da implantação de sistemas de manufatura customizados. O índice proposto permite estabelecer, para um dado produto de interesse, um ranking de customização para as suas características.

Tabela 6 - Comparação entre a priorização dos elementos de customização, considerando o $I C D_{i}^{*}$ e o $I C D 2_{i}$

\begin{tabular}{|c|c|c|c|c|}
\hline \multirow{2}{*}{ Item } & \multirow{2}{*}{ Elementos a serem customizados } & \multicolumn{2}{|c|}{ Priorização através do } & \multirow{2}{*}{ Diferença no ranking } \\
\hline & & $I C D_{i}^{*}$ & $I C D 2_{i}$ & \\
\hline 1 & Tipo de Eficiência do aparelho & 3 & 16 & -13 \\
\hline 2 & Escolha do nível de ruído & 1 & 6 & -5 \\
\hline 3 & Escolha da Tensão & 15 & 8 & 7 \\
\hline 4 & Escolha da Freqüência & 16 & 18 & -2 \\
\hline 5 & Escolha do tipo de Circulação de Ar & 20 & 9 & 11 \\
\hline 6 & Escolha da Versão (quente/frio) & 6 & 5 & 1 \\
\hline 7 & Tipo de controle & 5 & 17 & -12 \\
\hline 8 & Opção por kit de tratamento do ar & 11 & 3 & 8 \\
\hline 9 & Tipo de Gás & 19 & 7 & 12 \\
\hline : & : & : & : & : \\
\hline 20 & Escolha da Embalagem & 7 & 4 & 3 \\
\hline
\end{tabular}

ideais do processo de produção para a customização terão sido alcançadas;

- Os elementos de customização tipo de eficiência do aparelho e escolha da marca foram identificados como os mais prejudicados pelas condições de produção atuais. Estes elementos têm uma perda de 13 colocações na escala de priorização, como pode ser visto na Tabela 6. Como estes elementos são identificados pelos clientes como prioritários, são necessárias modificações na estrutura de produção do equipamento a fim de possibilitar a sua customização. Esta análise pode ser estendida aos demais elementos apresentados na Tabela 6;

- O elemento de customização tipo de gás foi identificado como o mais beneficiado pelas condições de produção existentes. Este elemento tem um ganho de 12 colocações na escala de priorização, como pode ser visto na Tabela 6 . Porém, tal elemento não é identificado pelos clientes como prioritário para a customização.
Na composição do índice de customização, três indicadores foram considerados: (i) demanda por customização estabelecida pelos usuários do produto relativamente às suas características, $(i i)$ flexibilidade dos fornecedores das matérias-primas que compõem o produto, e (iii) flexibilidade do processo de manufatura do produto.

Um estudo de caso no setor de eletrodomésticos ilustrou os passos metodológicos propostos. Os resultados permitiram verificar a consistência do índice proposto, já que elementos de customização demandada com baixa flexibilidade tiveram seus índices de importância deflacionados.

\section{Referências}

ADAMIDES, E. D. Responsibility-based manufacturing. Int. J. of Adv. Manufacturing Tech., v.11, p. 439-448, 1996. 
AKAO, Y. Quality Function Deployment: Integrating customer requirements into product design.

Cambridge: Productivity Press, 369p., 1990.

ATKINSON, J. Flexibility: Planning for an uncertain future. Manpower Policy and Practice. v.1, p.26-9, 1985.

BARAD, M.; NOF, S. Y. CIM flexibility measures: A review and a framework for analysis and applicability assessment. International Journal Computer Integrated Manufacturing, v.10, n.1-4, p.296-308, 1997.

BENSON, J. Management Strategy and labour flexibility in Japanese manufacturing enterprises. Human Res. Manage. J., v. 6, p.44-57, 1996.

BOYNTON, A. C.; VICTOR, B.; PINE II, B. J. New competitive strategies: Challenges to organizations and information technology. IBM Systems J., v.32, p.40-64, 1993.

BROWNE, J.; DUBOIS, D.; RATHMILL, K.; SETHI, S. P.; STECKE, K. E. Classification of flexible manufacturing systems. The FMS Mag., v.2, p.114-117, 1984.

CARTER, M. F. Designing flexibility into automated manufacturing systems. Proceedings, Second ORSA/ TIMS Special Conference on FMS, Ann Arbour, MI, p.107-118, 1986.

CHATTERJEE, A.; COEHN, M. A.; MAXWELL, W. L.; MILLER, L. L. Manufacturing flexibility: Models and measurements. Proceedings, First ORSA/TIMS Special Conference on Flexible Manufacturing Systems, Ann Arbour, MI., p.49-64, 1984.

CHUNG, C. H.; CHEN, I. J. A Systematic assessment of the value of flexibility from FMS. Proceedings, Third ORSA/TIMS Special Conference on FMS, New York, p.27-32, 1989.

CHURCHILL Jr., G. A.; PETER, J. P. Marketing: criando valor para o cliente. São Paulo: Saraiva, 2000.

COX Jr., T. Toward the measurement of manufacturing flexibility. Prod. and Inventory Manage. J., v.30, p.68-72, 1989.

CSILlAG, J. M. Análise do Valor. São Paulo: Atlas, 1995.

DA SILVEIRA, G.; BOREINSTEIN, D.; FOGLIATTO, F. S. Mass customization: Literature review and research directions. Int. J. of Prod. Econ., v.72, p.1-13, 2001.

DAVIS, S. M. "From future perfect": Mass Customizing. Planning Review, v.17, p.16-21, 1989.
DE TONI, A.; TONCHIA, S. Manufacturing flexibility: A literature review. Int. J. of Prod. Res., v.36, p.1587$1617,1998$.

DIXON, J. R. Measuring manufacturing flexibility: An empirical investigation. Eur. J. of Operational Res., v.60, p.131-143, 1992.

EASTWOOD, M. A. Implementing mass customization. Computer in Industry, v.30, n.3, p.171-174, 1996.

GERWIN, D. An agenda for research on the flexibility of manufacturing processes. Int. J. of Oper. and Prod. Manage, v.7, p.38-49, 1987.

GUPTA, Y. P.; GOYAL, S. Flexibility of manufacturing systems: Concepts and measurements. Eur. J. of Operational Res., v.43, p.119-135, 1989.

HART, C. W. Mass customization: Conceptual underpinnings, opportunities and limits. Int. J. of Service Ind. Manage, v.6, p.36-45, 1995.

HAYES, B. E. Measuring Customer Satisfaction: survey design, use, and statistical analysis methods. $2^{\mathrm{a}}$ ed., Milwaukee-USA: American Society for Quality, 1998.

HEIJLTJES, M. G. Advanced manufacturing technologies and HRM policies, findings from chemical and drink companies in the Netherlands and Great Britain. Organ. Stud., v.21, p.775-805, 2000.

JAIKUMAR, R. Flexible manufacturing systems: A managerial perspective. Working Paper, Harvard Business School, Boston, MA, 1984.

JIAO, J.; TSENG, M. M. Understanding product family for mass customization by developing commonality indices. J. of Eng. Design, v.11, p.225-243, 2000.

KAY, M. Making mass customization happen: Lessons for implementation. Planning Rev., v.21, p.14-18, 1993.

KOTHA, S. Mass customization: Implementing the emerging paradigm for competitive advantage. Strategic Manage. J., v.16, p.21-42, 1995.

KOTHA, S. From mass production to mass customization: The case of the National Industrial Bicycle Company of Japan. European Management Journal, v.14, n.5, p.442-450, 1996.

KOTLER, P. Administração de marketing: análise, planejamento, implementação e controle. $5^{\mathrm{a}}$ ed., São Paulo: Atlas, 1998. 
LAMPEL, J.; MINTZBERG, H. Customizing customization. Sloan Manage. Rev., v.38, p.21-30, 1996.

LAU, R. Mass customization: the next industrial revolution. Industrial Management, v.37, n.5, p.18-19, 1995.

LEI, D.; HITT, M. A.; GOLDHAR, J. D. Advanced manufacturing technology: Organizational design and strategic flexibility. Organ. Stud., v.17, p.501-523, 1996.

LIECHTY, J.; RAMASWAMY, V.; COHEN, S. H. Choice menus for mass customization: An experimental approach for analyzing customer demand with an application to a web-based information service. J. of Marketing Res., v.38, p.183-196, 2001.

McCUTCHEON, D. M.; RATURI, A. S.; MEREDITH, J. M. The customization-responsiveness squeeze. Sloan Manage. Rev., v.35, p.89-99, 1994.

MURY, L.G.M. Uma metodologia para adaptação e melhoria de produtos a partir da engenharia reversa. Dissertação de Mestrado, Programa de Pós-Graduação em Engenharia de Produção, UFRGS. Porto Alegre, 2000.

NARASIMHAN, R.; DAS, A. An empirical investigation of the contribution of strategic sourcing to manufacturing flexibilities and performance. Decisions Sciences, v.30, n.3, p.683-718, 1999.

NEWMAN, W. R.; HANNA, M.; MAFFEI, M. J. Dealing with the uncertainties of manufacturing: Flexibility, buffers and integration. Int. J. of Oper. and Prod. Manage., v.13, p.19-34, 1993.

OLHAGER, J. Manufacturing flexibility and profitability. International journal of production economics, v.30-31, p.67-78, 1993.

PARKER, R. P.; WIRTH, A. Manufacturing flexibility: Measures and relationships. European Journal of Operational Research, v.118, p.429-449, 1999.

PINE II, B. J.; PIETROCINI, T. W. Standard modules allow mass customization at Bally Engineered Structures. Planning Review, v.21, n.4, p.20-22, 1993.

RAMASESH, R. V.; JAYAKUMAR, M. D.

Measurement of manufacturing flexibility: A valuebased approach. Journal of Operations Management, v.10, n.4, 1991.
ROSA, J. A. Roteiro prático para desenvolvimento de novos produtos. São Paulo: Editora STS, 1999.

SETHI, A. K.; SETHI, S. P. Flexibility in manufacturing: A survey. The International Journal of Flexible Manufacturing Systems, v.2, p.289-328, 1990.

SHEWCHUK, J. P.; MOODIE, C. L. Definition and classification of manufacturing flexibility types and measures. The International Journal of Flexible Manufacturing Systems, v.10, p.325-349, 1998.

SLACK, N. The flexibility of manufacturing systems. Int. J. of Oper. and Prod. Manage., v.7, p.35-45, 1987.

SLACK, N.; CORRÊA, H. The flexibilities of push and pull. Int. J. of Oper. and Prod. Manage., v.12, p.82-92, 1992.

SLACK, N. Vantagem Competitiva em manufatura: atingindo competitividade nas operações industriais. São Paulo: Atlas, 198p., 1993.

SONG, L.; NAGI, R. Design and implementation of a virtual information system for agile manufacturing. IIE Trans., v.29, p.839-857, 1997.

SOPHIE-LEE, C. H.; BARUA, A.; WHINSTON, A. B. The complementarity of mass customization and electronic commerce. Economics of Innovation and New Technology, v.9, p.81-109, 2000.

UPTON, D. M. The management of manufacturing flexibility. California Manage. Rev., v.36, p.72-89, 1994.

VAN DIJK, M. Flexible specialization, the new competition and industrial districts. Small Bus. Econ., v.7,p.15-27, 1995

VAN HOEK, R. I. The role of third-party logistics providers in mass customization. Int. J. of Logistics Manage., v.11, p.37-46, 2000.

WESTBROOK, R.; WILLIAMSON, P. Mass customization: Japan's new frontier. Eur. Manage. J., v.11, p.38-45, 1993.

ZIPKIN, P. The limits of mass customization. MIT Sloan Manage. Rev., v.42, p.81-87, 2001.

Submetido em outubro/2001

Aprovado em fevereiro/2002 